\title{
Novedades para el catálogo briológico de Navarra (norte de España)
}

\author{
Alicia Ederra, Noemí Iglesias, Ruth Juaristi y Beatriz Huarte ${ }^{1}$
}

\begin{abstract}
Resumen: Ederra, A.; Iglesias, N.; Juaristi, R. \& Huarte, B. 2012. Novedades para el catálogo briológico de Navarra (norte de España). Bot. Complut. 36: 35-38.

Se añaden al catálogo briológico de Navarra 13 especies de musgos, aportando datos de su distribución en Navarra y en España.

Palabras clave: musgos, área de distribución, especies amenazadas, Península Ibérica.

Abstract: Ederra, A.; Iglesias, N.; Juaristi, R. \& Huarte, B. 2012. New records for the bryological checklist of Navarra (North Spain). Bot. Complut. 36: 35-38.
\end{abstract}

Thirteen species are added to the checklist of mosses of Navarra, giving information about their distribution areas in Navarra and Spain.

Key words: mosses, distribution area, threatened species, Iberian Peninsula.

\section{INTRODUCCIÓN}

La brioflora de Navarra (norte de España) ha sido estudiada desde antiguo (Lacoizqueta 1885) y es una de las mejor conocidas de la Península Ibérica. Además es una de las más ricas, seguramente debido a que, por su localización geográfica, en Navarra se encuentran las fronteras entre el mundo atlántico, el mediterráneo y el pirenaico, 10 que se traduce en la existencia de gran diversidad de bosques, paisajes y hábitats (Fig. 1). Sin embargo, muestreos realizados en la última década han arrojado como resultado varias decenas de novedades a añadir al catálogo briológico de Navarra. Así, en Huarte Irurzun (2001) se elevó el número de musgos de Navarra a 466 y en Juaristi Iranzo (2005) se añadieron otros 17. Otras prospecciones han dado lugar a algunas novedades más, con lo que actualmente el catálogo de musgos de Navarra comprende 490 taxones.

Un buen conocimiento de la flora de una región es fundamental a la hora de promover planes de gestión y conservación del territorio y de su biodiversidad, así como de establecer con base adecuada la catalogación de las especies según grados de amenaza. Por ejemplo, en Sérgio et al. (2006) hay 116 especies de musgos catalogadas como DD, DD-new y DD-vanished, es decir, con información inade- cuada acerca de su distribución o no bien conocidas a nivel taxonómico, lo cual impide su correcta evaluación en las diferentes categorías de amenaza. Esto quiere decir que toda aportación que permita conocer mejor las áreas de distribución será útil para poder catalogar las especies e implementar medidas de protección o conservación.

En este trabajo aportamos datos de 13 especies que, además de resultar novedades para el catálogo de musgos de Navarra, son interesantes a nivel peninsular por su corología o por su estatus a nivel de amenaza.

\section{RESULTADOS}

Presentamos los taxones por orden alfabético, siguiendo la nomenclatura de Hill et al. (2006). Las muestras están depositadas en el herbario de la Universidad de Navarra (PAMP), con los números que se indica en cada especie.

\section{Dicranum majus Sm. (Fig. 2)}

NavarRa: Elizondo, 30TXN27, PAMP 7814. Terrícola en robledal de Quercus robur.

\footnotetext{
${ }^{1}$ Departamento de Biología Vegetal, sección Botánica, Facultad de Ciencias, Universidad de Navarra. C/ Irunlarrea s/n, 31008 Pamplona, España.aederra@unav.es,niglesias@alumni.unav.es,rjuaira@alumni.unav.es,bhuarte@unav.es

Recibido: 7 marzo 2012. Aceptado: 17 abril 2012.
} 


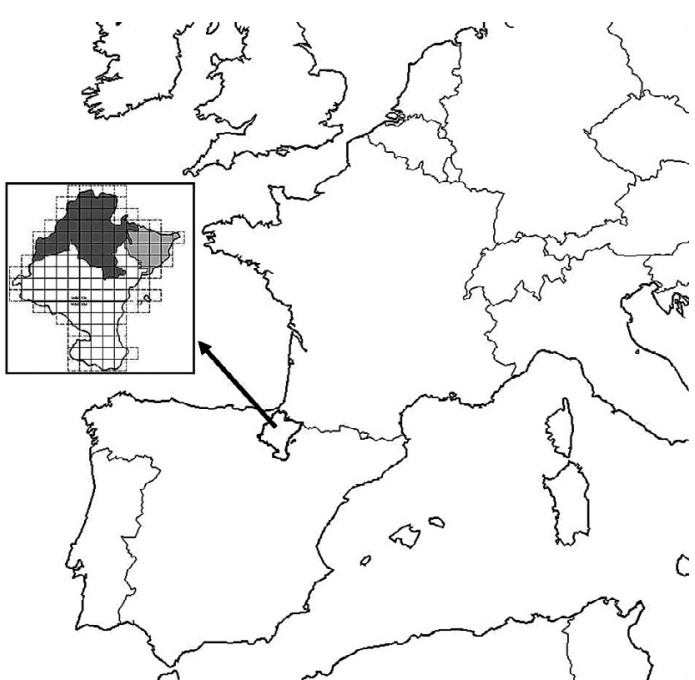

Fig. 1- Situación de Navarra. En gris oscuro, zona atlántica; en gris claro, zona pirenaica; en blanco, zona mediterránea.

Especie poco citada en España, se conoce de Asturias (Fernández Ordóñez 1981; Fernández Ordóñez et al. 2003), de Cantabria (Oliván et al. 2003) y del País Vasco (Allorge 1955; Infante Sánchez \& Heras Pérez 2008); la cita de Zubía Icazuriaga (1921) no ha podido ser confirmada (Martínez Abáigar \& Núñez Olivera 1996). La 10calidad navarra marca el límite oriental de su área de distribución en España. Sergio et al. (2006) catalogan D. majus como LC (Preocupación menor). Sin embargo, las citas de esta especie son pocas y las recolecciones antiguas, ya que incluso la que aquí publicamos como novedad data ya de 1995 .

\section{Fontinalis hypnoides C. Hartm. (Fig. 2)}

NAVARRa: Usún: Foz de Arbayún, 30TXN5624, PAMP 9808; Fitero: Hoya del Puente, 30TWM95, al borde del río, PAMP 9807. Epífita y saxícola, en acequias y árboles de bordes de río y sumergida.

Según Guerra (2012a) se encuentra por toda la península, siendo algo más rara hacia el centro y el norte.

Grimmia tergestina Bruch \& Schimp. (Fig. 2)

Navarra: Carretera Cintruénigo-Cascante, 30TXM05, PAMP 9809; Carretera Tafalla-Falces, 30TXM09, PAMP 9810; Ujué: Sierra de Ujué, 30TXM2699, PAMP 9837; Ulzúrrun: Alto de Ulzúrrun, 30TWN94, PAMP 9812.

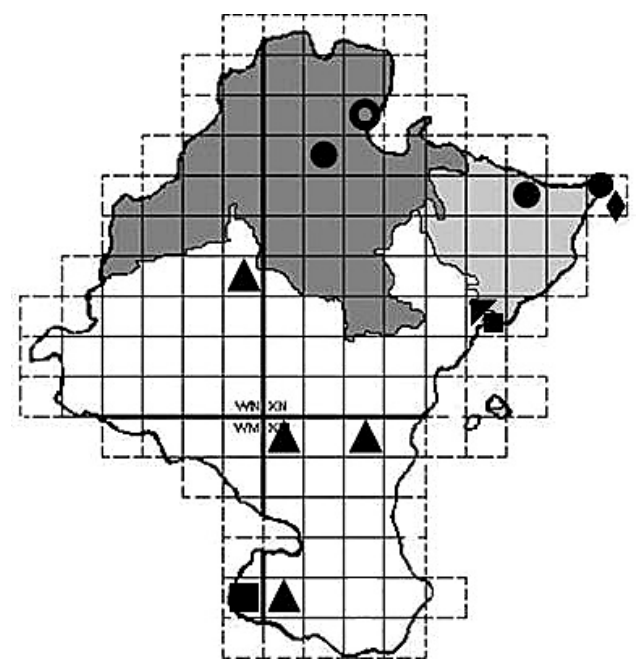

Fig. 2- Localización en Navarra de Dicranum majus (ani1lo), Fontinalis hypnoides (cuadrado), Grimmia tergestina (triángulo equilátero), Lescuraea saxicola (rombo), Orthotrichum alpestre (círculo), y Orthotrichum sprucei (triángulo rectángulo).

Especie bastante común hacia el sur y el levante español, conocida también de Cantabria (Fuertes et al. 1998).

\section{Lescuraea saxicola (Schimp.) Molendo (Fig. 2)}

NAVARRA: Isaba: Larra, 30TXN8059, PAMP 9813. Saxícola en hayedo eutrofo de la asociación Scillo lilio-hyacinthi-Fagetum sylvaticae, a $1600 \mathrm{~m}$.

Conocida de Lérida y Huesca (Casas et al. 1995; Infante et al. 2002), la presente cita marca el límite occidental de su distribución en España.

\section{Neckera besseri (Lobarz.) Jur. (Fig. 3)}

NaVarRa: Uztárroz, 30TXN6751, 950 m, PAMP 9501; Isaba: Puerto de los Navarros, 30TXN7748, 1500 m, PAMP 9814. Epífito en boj y en haya, siempre dentro de bosques, bien de Quercus humilis, bien de Fagus sylvatica y Pinus sylvestris.

Según Guerra (2012b) las localidades que aportamos marcan el límite noroccidental de distribución en España.

Orthotrichum alpestre Bruch \& Schimp. (Fig. 2)

NaVARRA: Puerto de Velate, 30TXN16, 900 m, PAMP 1861; Isaba: Larra, 30TXN8059, 1600 m, PAMP 9816; Uztárroz: Fuente Urona, 30TXN6751, 900 m, PAMP 9815. Siempre epífita en haya. 


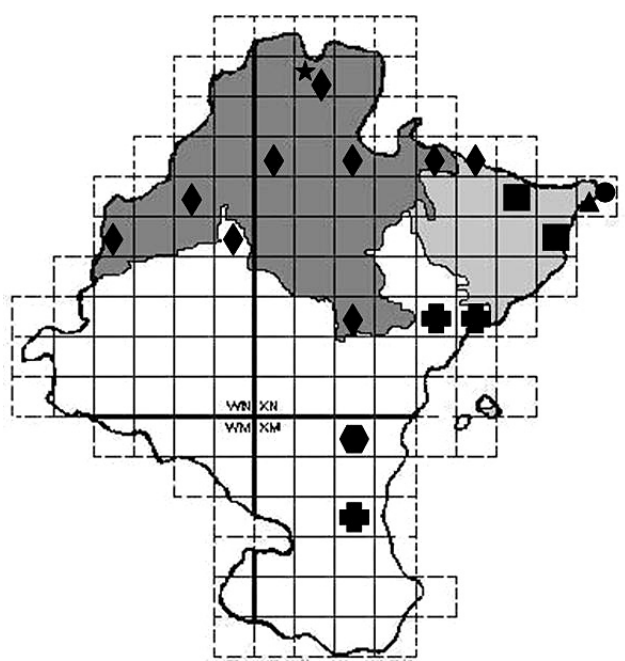

Fig. 3- Localización en Navarra de Neckera besseri (cuadrado), Paraleucobryum sauteri (triángulo), Phascum vlasovii (hexágono), Pohlia andalusica (estrella), Pseudoleskeella nervosa (círculo), Syntrichia ruralis var. ruraliformis (cruz) y Trichodon cylindricus (rombo).

\section{Orthotrichum sprucei Mont. (Fig. 2)}

NavarRa: Usún: Foz de Arbayún, 30TXN5624, PAMP 9817. Epífito en fresnos en un bosquete de sauces y fresnos al borde del río.

Esta localidad marca el límite oriental de distribución en España (Lara et al. 2006). En Sérgio et al. (2006) figura como DD.

\section{Paraleucobryum sauteri (Bruch \& Schimp.)} Loeske (Fig. 3)

NaVARRA: Isaba: Larra, 30TXN8059, 1600 m, PAMP 9818 y PAMP 9819. Epífita en haya.

En España se conoce sólo de los Pirineos, y esta localidad marca el límite occidental de distribución en nuestro país. Sérgio et al. (2006) la consideran VU.

\section{Phascum vlassovii Laz. (Fig. 3)}

NavarRa: Monasterio de la Oliva, 30TXM2692, PAMP 9820. Terrícola en jardín sombrío.

Guerra (2006) la cita de Navarra sin indicar localidades; dado que en Sérgio et al. (2006) se considera VU, nos parece importante dar a conocer la localidad precisa.
Pohlia andalusica (Höhn.) Broth. (Fig. 3)

NaVARRA: Señorío de Bértiz, 30TXN18, PAMP 9821. Apareció en un cultivo de suelo recolectado en un hayedo.

Guerra (2010) la ha dado a conocer de Granada, Huesca, Lérida y también Navarra, sin indicar la localidad. Sérgio et al. (2006) la catalogan como NT (casi amenazada) y por eso aportamos aquí el detalle de su situación. No obstante, dado que sólo ha aparecido en un cultivo, y nunca de forma espontánea en el bosque, su consideración como taxón propio de la brioflora de Navarra puede ser discutible.

Pseudoleskeella nervosa (Brid.) Nyholm (Fig. 3)

NAVARRA: Isaba: Larra (30TXN8059), 1600 m, PAMP 9822. Saxícola en hayedo eutrofo de la asociación Scillo lilio-hyacinthi-Fagetum sylvaticae.

Esta localidad es la más occidental en los Pirineos (Brugués \& Muñoz 1999, Casas et al. 2006).

\section{Syntrichia ruralis (Hedw.) F. Weber \& D. Mohr} var. ruraliformis (Besch.) Delogne (Fig. 3)

NaVARRA: Yesa: Sierra de Leyre, 30TXN5721, 650 m, PAMP 9823; Lumbier: Alto de Arangoiti, 30TXN4723, 1300 m, PAMP 9824 y PAMP 9825; Bardena Blanca, 30TXM27, PAMP 9826. Terrícola y saxícola en matorrales, pastizales y coscojares secos y pedregosos.

Se trata de un taxón bastante común en la Península Ibérica que, sin embargo, todavía no se había citado de Navarra.

Trichodon cylindricus (Hedw.) Schimp. (Fig. 3)

NaVARRA: Sierra de Izco, 30TXN22, PAMP 9841; Señorío de Bértiz, 30TXN18, PAMP 9842; Sierra de Urbasa, 30TWN64, PAMP 9843; Oroquieta, 30TXN06, PAMP 9844; Quinto Real, 30TXN26, PAMP 9831; Echauri, 30TWN94, PAMP 9832; Sierra de Aralar, 30TWN85, PAMP 9833; Selva de Irati, 30TXN46 y 30TXN56, PAMP 9834 y PAMP 9835 respectivamente. En todos los casos se ha encontrado en cultivos de suelos recolectados en hayedos.

Según Puche (2012) es una especie que vive en suelos ácidos, descubiertos, bordes de caminos y taludes, en las provincias de Alicante, Barcelona, Gerona, Lérida, Navarra (sin localidades concretas) y Guipúzcoa. Según Sérgio et al. (2006) es VU (vulnerable), por lo que nos pare- 
ce útil aportar estos datos. Sin embargo, como en el caso de Pohlia andalusica, puede discutirse su consideración como especie propia de la brioflora de Navarra, aunque su participación del banco de diásporas de los hayedos queda fuera de toda duda.

\section{BIBLIOGRAFÍA}

Allorge, V. 1955. Catalogue préliminaire des muscinées du Pays basque français et espagnol. Revue. Bryol. Lichénol. 24: 96-333.

BruguÉs, M. \& MuÑoz, J. 1999. Clave para la determinación de las Leskeáceas de España. Bol. Soc. Esp. Briol. 15: 11-14.

Casas, C.; Brugués, M. \& Cros, R. M. 1993. Els briòfits del Parc Nacional d'Aigüestortes i Estany de Sant Maurici i la seva zona d'influència. La investigació al Parc Nacional d'Aigüestortes i Estany de Sant Maurici 2: 73-81.

Casas, C.; Cros, R. M.; Brugués, M.; Ruíz, E.; Sérgio, C.; BARRÓN, A. \& LLORET, F. 2006. Aportaciones a la brioflora del Pirineo. Bol. Soc. Esp. Briol. 28: 73-86.

FERNÁNDEZ ORdóÑEZ, M. C. 1981. Estudio de la flora briológica del Valle del Nalón y Puerto de Tarna. Bol. C. Nat. IDEA 28: 43-218.

Fernández Ordóñez, M. C.; Díaz González, T. E. \& CollaDo Prieto, M. A. 2003. Evaluación de la biodiversidad briopteridofítica de la cuenca del río de Nueva (Llanes, Asturias, N Península Ibérica): Bases para su protección y conservación. Acta Bot. Barc. 49: 191-208.

Fuertes, E.; Acón, M.; Infante, M. \& Oliva, R. 1998. Aportaciones a la flora briólogica española. Notula XII. Musgos y hepáticas de La Liébana (Cantabria, España). Lazaroa 19: 43-55.

GuerRA, J. 2006. Phascum Hedw. En J. Guerra, M. J. Cano \& R. M. Ros (Eds.), Flora Briofitica Ibérica, 3: Pottiales, Encalyptales: 176-180. Universidad de Murcia y Sociedad Española de Briología.

GuerRA, J. 2010. Pohlia Hedw. En J. Guerra, M. Brugués, M. J. Cano \& R. M. Cros (Eds.), Flora Briofítica Ibérica, 4: Funariales, Splachnales, Schistostegales, Bryales, Timmiales: 183-206. Universidad de Murcia y Sociedad Española de Briología.

GUERRA, J. 2012a. Fontinalaceae Schimp. www.florabriofiticaiberica.com/borradores. Acceso 28/02/2012.

Guerra, J. 2012b. Neckera Hedw. www.florabriofiticaiberica.com/borradores. Acceso 28/02/2012.

Hill, M. O.; Bell, N.; Bruggeman-Nannenga, M. A.; BruguÉs, M.; CAno, M. J.; Enroth, J.; Flatberg, K. I.; Frahm, J.-P.; Gallego, M. T.; Garilleti, R.; GuerRA, J.; Hedenäs, L.; Holyoak, D. T.; Hyvönen, J.; Ignatov, M. S.; Lara, F.;
MAZIMPAKA, V.; MuÑoz, J. \& SÖDERSTRÖM, L. 2006. An annotated checklist of the mosses of Europe and Macaronesia. J. Bryol. 28: 198-267.

HuARTE IruRZun, B. 2001. Estudio de la flora muscinal de Navarra: actualización, profundización, catálogo y análisis. Tesis Doctoral inédita. Universidad de Navarra.

InFANTE SÁnchez, M. \& Heras Pérez, F. 2008. Briófitos del hayedo de Oieleku. Seguimiento de comunidades epifíticas y lignícolas. Actualizaciones. Dirección de Montes y Medio Natural, Departamento para el Desarrollo del Medio Rural. Diputación Foral de Gipuzkoa.

Infante, M.; Segarra, J. G. \& Heras, P. 2002. Preliminary checklist of the bryophytes of Aragón (Spain). Flora Mediterranea 12: 183-259.

Juaristi IRAnzo, R. 2005. Estudio de la flora muscinal del Pirineo Occidental. Tesis Doctoral inédita. Universidad de Navarra.

LACOIZQUETA, J. M. 1885. Catálogo de las plantas que espontáneamente crecen en el Valle de Bertizarana. An. Soc. Esp. Hist. Nat. 14: 192-204.

Lara, F.; Garilleti, R.; Albertos, B.; Medina, R. \& MazimPAKA, V. 2006. Nuevos datos sobre la distribución en la Península Ibérica de Orthotrichum sprucei Mont., O. rivulare Turn. y Dendrocryphaea lamyana (Mont.) P. Rao. Bol. Soc. Esp. Briol. 28: 61-66.

MartíneZ-ABaigar, J. \& NúÑEz-Olivera, E. 1996. The bryological work of Ildefonso Zubía Icazuriaga (1819-1891) in northern Spain. Nova Hedwigia 62 (1-2): 255-266.

Oliván, G.; FuERTES, E. \& ACón, M. 2003. Revisión y estado de conservación de la flora briológica de la Sierra de Peña Sagra (Cordillera Cantábrica, España). Acta Bot. Barc. 48: 183-189.

Puche, F. 2012. Género Trichodon. www.florabriofiticaiberica.com/borradores. Acceso 28/02/2012.

Sérgio, C.; Brugués, M.; Cros, R. M.; Casas, C. \& García, C. 2006. The 2006 Red List and an updated Check List of Bryophytes of the Iberian Peninsula (Portugal, Spain and Andorra). Lindbergia 31: 109-126.

ZuBía ICAZURIAGA, I. 1921. Reseña de la provincia de Logroño como preliminar al conocimiento de la Flora de la Rioja. Imprenta y Librería Moderna, Logroño. 